\title{
Validation of a CFD model for flow in meandering rivers using an experimental test-setup: first results
}

\author{
K. Delecluyse ${ }^{1}$, W. Brantegem ${ }^{1}$, P. Troch ${ }^{1}$, R. Verhoeven ${ }^{1}$ \\ \& J. Vierendeels ${ }^{2}$ \\ ${ }^{1}$ Laboratory of Hydraulics, Department of Civil Engineering, \\ Ghent University, Ghent, Belgium \\ ${ }^{2}$ Department of Flow, Heat and Combustion Mechanics, \\ Ghent University, Ghent, Belgium
}

\begin{abstract}
A 3D Reynolds Averaged Navier Stokes (RANS) model of a meandering channel with rectangular cross-section has been developed using the commercial software package FLUENT 6.2. This model solves the 3D Navier-Stokes equations using the PISO scheme for the pressure-velocity coupling and the realizable k- $\varepsilon$ model for turbulence closure. Output of the numerical model is compared to validation experiments conducted in a physical model, which represents two wavelengths of a regime channel and allows for the measurement of flow patterns at several discharges and variable bed forms. The computed water depths and velocity profiles of the CFD model output are in good agreement with the physical model results. The simulations slightly underpredict the streamwise velocities, which reach a maximum just before the apex of the meander bend, at the inner bank in the lower part of the flow depth. The CFD model also captures the motion of the secondary current or transverse flow well, showing the same direction of current along the entire second wavelength.
\end{abstract}

Keywords: CFD, numerical model, meandering rivers, open channel flow, experimental results. 


\section{Introduction}

During the last century, a large number of rivers have been artificially straightened in order to improve the manoeuvrability of ships, ease the drainage of rain water and make more space for expanding urban areas. These measures however brought along a number of drawbacks. Nowadays, the trend in river management is to give the river back its former space and to restore its natural flow and meandering pattern.

In order to accurately predict this remeandering process of artificially straightened rivers, a detailed knowledge of the flow characteristics and sediment transport processes in meandering river bends is required. The study of two- and three-dimensional flow in open channels has recently experienced a surge of interest in the application of computational fluid dynamics (CFD) to hydrological and geomorphological problems, showing great potential in those problems where the spatio-temporal boundary conditions may vary over a wide range, thus often making field study either impracticable or impossible.

In this paper a 3D CFD model is presented to investigate and predict the flow in a meandering river. To validate the numerical model, an experimental model was built which represents two wavelengths of a regime channel. Previous studies have shown that combining the numerical models with a physical model is a useful method to validate the numerical output, although it can be difficult to trace whether any differences between the two are caused by numerical simplifications or experimental errors (e.g. Olsen and Kjellesvig [1]; Rajendran et al [2]). A first set of experiments with rectangular, fixed cross-section and steady discharge rate was performed, and the results are presented in this paper. Both the experimental and numerical results are compared to the theory of flow in a meandering river, and discussed in detail.

\section{Experimental model}

The experimental model shown in Figure 1 represents two wavelengths of a regime channel, the dimensions of which were determined according to Williams [3]. At the inlet, the discharge is determined by means of a calibrated triangular weir. Before entering the channel geometry, the water flows through a series of parallel tubes, to ensure a uniform velocity profile. At the outlet, the water flows over a rectangular weir with adjustable height, and is collected in a container after which the water gets pumped into the inlet construction again, thus forming a closed circuit. The sides and bottom of the entire second wavelength are made out of a transparent material to allow for the future use of Particle Image Velocimetry (PIV) measurement techniques. In the early stages of the research, this model is solely used to measure velocity profiles in a meander bend, without incorporating sediment transport processes. For this reason, the rectangular cross section and the discharge remain constant during the experiment. Later on, when the sediment transport module will be introduced into the numerical model, the experimental model will be adjusted to enable variable cross-sections and discharges. 


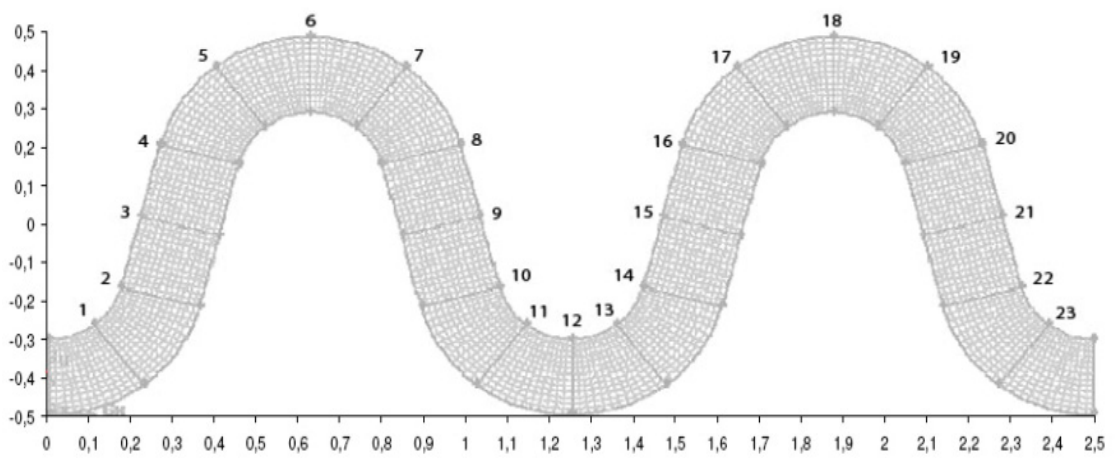

Figure 1: Geometry and computational grid for the experimental and numerical models. Indicated dimensions are in [m]. The numbers refer to the cross-sections in which velocity measurements were performed.

\section{Hydrodynamic model}

\subsection{Model equations}

The flow field is determined by the following Reynolds-averaged Navier-Stokes (RANS) and continuity equations, written in cartesian coordinates:

$$
\begin{gathered}
\frac{\partial u_{i}}{\partial x_{i}}=0 \\
\frac{\partial u_{i}}{\partial t}+\frac{\partial\left(u_{i} u_{j}\right)}{\partial x_{i}}=-\frac{1}{\rho} \frac{\partial p}{\partial x_{i}}+\frac{1}{\rho} \frac{\partial \tau_{i j}}{\partial x_{j}}+F_{i}
\end{gathered}
$$

where $u_{i}(\mathrm{i}=1,2,3)$ are the velocity components; $F_{i}$ is the gravity force per unit volume; $\rho=$ fluid density and $p=$ pressure. The effective shear stresses $\tau_{i j}$ are calculated with the k- $\varepsilon$ turbulence model (Rodi [4]), which employs the eddy viscosity relation

with

$$
\tau_{i j}=\rho\left(v+v_{t}\right)\left(\frac{\partial u_{i}}{\partial x_{j}}+\frac{\partial u_{j}}{\partial x_{i}}\right)-\frac{2}{3} \delta_{i j} k
$$

$$
v_{t}=c_{\mu} k^{2} / \varepsilon
$$

where the turbulent kinetic energy $\mathrm{k}$ and its dissipation rate $\varepsilon$ determining the eddy viscosity $v_{\mathrm{t}}$ are obtained from the following model equations:

with

$$
\begin{gathered}
\frac{\partial k}{\partial t}+\frac{\partial\left(u_{j} k\right)}{\partial x_{j}}=\frac{\partial}{\partial x_{j}}\left(\frac{v_{t}}{\sigma_{k}} \frac{\partial k}{\partial x_{j}}\right)+G-\varepsilon \\
\frac{\partial \varepsilon}{\partial t}+\frac{\partial(\varepsilon)}{\partial x_{j}}=\frac{\partial}{\partial x_{j}}\left(\frac{v_{t}}{\sigma_{\varepsilon}} \frac{\partial \varepsilon}{\partial x_{j}}\right)+\left(c_{\varepsilon 1} G-c_{\varepsilon 2} \varepsilon\right) \frac{\varepsilon}{k}
\end{gathered}
$$

$$
G=v_{t}\left(\frac{\partial u_{i}}{\partial x_{j}}+\frac{\partial u_{j}}{\partial x_{i}}\right) \frac{\partial u_{i}}{\partial x_{j}}
$$


the production of $\mathrm{k}$. The following values of the model coefficients are used: $\mathrm{c}_{\mu}=$ $0.09 ; \mathrm{c}_{\varepsilon 1}=1.44 ; \mathrm{c}_{\varepsilon 2}=1.9 ; \sigma_{\mathrm{k}}=1$ and $\sigma_{\varepsilon}=1.2$.

\subsection{Water depth calculations}

In this paper, the Volume of Fluid Technique (VOF, Hirt and Nichols [5]) has been incorporated into the solution of the Navier-Stokes equations in order to predict the water surface elevation, as opposed to a 'fixed lid approach'. For use of the latter, detailed knowledge of the river bathymetry is absolutely necessary, as any incorrect 'fixed lid' will affect the distribution of the mass and momentum of the fluid flow in the numerical simulation, leading to errors in the prediction of bed shear stress, and consequently, bed load transport (Ma et al [6]). Although the model presented in this paper does not yet include a sediment transport module, such a module will be implemented in the near future. Therefore, the VOF technique was selected for water depth calculations.

Using the VOF technique, the flow consists of two phases: water and air. The volume fraction of the water, $\mathrm{F}$, is introduced in each computational cell and is defined as follows:

$$
F=\frac{\delta \Omega_{\text {water }}}{\delta \Omega_{\text {cell }}}
$$

where $\delta \Omega_{\text {cell }}$ is the volume of the computational cell and $\delta \Omega_{\text {water }}$ is the fraction of the cell filled with water. Thus, the following applies:

$$
\left\{\begin{array}{c}
F=1, \text { cell is full of water } \\
F=0, \text { cell is full of air } \\
0<F<1, \text { cell is filled with water and air }
\end{array}\right.
$$

The governing fluid flow equations for the water flow and the air flow above the water are expressed in a single form as given by eqns. (1) and (2), but the physical properties that appear in eqns. (1) and (2) are different and are defined by the volume-fraction-weighted average of physical properties of the air and water as follows:

$$
\begin{aligned}
& \rho=F \rho_{\text {water }}+(1-F) \rho_{\text {air }} \\
& \mu=F \mu_{\text {water }}+(1-F) \mu_{\text {air }}
\end{aligned}
$$

According to the law of mass conservation of air and water, the volume fraction of the water satisfies

$$
\frac{\partial F}{\partial t}+u_{i} \frac{\partial F}{\partial x_{i}}=0
$$

By numerically solving the volume fraction eqn. (12), the volume fractions of water, F, and of air, 1-F, in a control volume cell may be obtained.

\subsection{Boundary conditions}

As upstream boundary condition, the velocity distribution for both phases and the water depth are given, consistent with the water discharge through the model. At the downstream end the water depth is specified, along with a zero gradient 
boundary condition. At the river bed, the standard wall function (Launder and Spalding [7]) is employed, given by:

$$
u^{+}=\frac{1}{\kappa} \ln \left(E y^{+}\right)-\Delta B\left(K_{s}^{+}\right)
$$

with $\mathrm{u}^{+}=\mathrm{u} / \mathrm{u}^{*}, \mathrm{y}^{+}=\mathrm{y} / \mathrm{y}^{*}, \mathrm{~K}_{\mathrm{s}}^{+}=\mathrm{K}_{\mathrm{s}} / \mathrm{y}^{*}, \mathrm{y}^{*}=\mathrm{v} / \mathrm{u}^{*}$ and $\mathrm{u}^{*}=\left(\tau_{\mathrm{w}} / \rho\right)^{1 / 2}$. In this, $\mathrm{u}$ is the fluid velocity parallel to the solid wall, $y$ is the distance from the wall, $\mathrm{u}^{*}$ is the wall friction velocity, $\mathrm{K}_{\mathrm{s}}$ is the wall roughness height, $\mathrm{E}$ is a constant taking a value of 9.8, $\Delta \mathrm{B}$ is an expression dealing with hydraulically rough beds, $\kappa$ is Von Karman's constant which usually takes a value of $0.4187, v$ is the dynamic viscosity of the fluid and $\tau_{\mathrm{w}}$ is the shear stress at the solid wall boundaries. In the study reported in this paper, a smooth bed and vertical banks have been employed in the model, so that $\Delta \mathrm{B}=0$. The turbulent kinetic energy $\mathrm{k}$ and dissipation rate $\varepsilon$ of the fluid flow at the upstream and downstream boundaries are determined by specifying the turbulent intensity of the fluid flow. In this paper a turbulent intensity of $10 \%$ was assumed for both upstream and downstream boundaries (Ma et al [6], Nallasamy [8]).

\subsection{Solution strategy}

Having set up the grid and specified the necessary boundary conditions, the governing fluid flow equations are solved by means of the control volume method. The Power Law scheme (Patankar [9]) is used for the space discretisation of the convection terms, while a second order implicit scheme is used for the discretisation of the transient terms. For pressure-velocity coupling, the PISO scheme is employed (Issa [10]). This scheme is part of the SIMPLE (Patankar [9]) family of algorithms and is based on the higher degree of the approximate relation between the corrections for pressure and velocity. The main idea of the PISO algorithm is to move the repeated calculations required by SIMPLE and SIMPLEC inside the solution stage of the pressure-correction equation. After one or more additional PISO loops, the corrected velocities satisfy the continuity and momentum equations more closely. The PISO algorithm takes a little more CPU time per solver iteration, but it can dramatically decrease the number of iterations required for convergence, especially for transient problems.

\section{Results}

In order to validate the numerical model, physical model experiments were conducted, along with several numerical experiments, in which the sensitivity of the computations to changes in model parameters was tested. The following section discusses the main results obtained from these experiments.

\subsection{Water depth}

A first experiment was conducted with the outlet height at $5 \mathrm{~cm}$, and a steady discharge $\mathrm{Q}$ of 2 1/s. Measurements along the experimental channel length show that the numerically computed water depths are a very accurate representation of 
the water depths in the physical model. Moreover, the water depth drops in the inner bends and rises in the outer bends, in agreement with the theory of flow through meander bends.

To investigate the influence of the discharge on the computed water depths, a second numerical experiment was conducted, with the discharge set at $2.5 \mathrm{l} / \mathrm{s}$. Because the type of weir at the outlet remains the same in all experiments, the numerical results can be validated by means of the calibration formula for the outlet weir

$$
Q=\frac{2}{3} C_{d} b h^{\frac{3}{2}} \sqrt{2 g}
$$

with Q the discharge, $\mathrm{b}$ the outlet width, $\mathrm{h}$ the water height above the outlet weir, $\mathrm{g}$ the gravitational acceleration and $\mathrm{C}_{\mathrm{d}}$ the dimensionless discharge coefficient. For the same type of outlet, the value of $\mathrm{C}_{\mathrm{d}}$ is a constant. For both experiments the value of $h$ was calculated by subtracting the height of the outlet weir from the computed water depth, and was substituted in eqn. (14). This yielded a $\mathrm{C}_{\mathrm{d}}$ of 0.822 for the first experiment and a $\mathrm{C}_{\mathrm{d}}$ of 0.812 for the second experiment. Thus, it can be concluded that the numerical model accurately predicts the water depths for any value of the discharge $\mathrm{Q}$.

In a third numerical experiment, the height of the outlet weir was changed from $5 \mathrm{~cm}$ to $6 \mathrm{~cm}$, with a discharge of 2 1/s. According to eqn. (14), the value of the overflow height $h$ should remain unaffected by this change. Calculation of $h$ for the first experiment yielded a value of $2.64 \mathrm{~cm}$, and a value of $2.58 \mathrm{~cm}$ for the third experiment. Thus, it can be concluded that the numerical model adapts very well to changes of the height of the outlet weir and calculates the water depths accordingly.

\subsection{Velocity profiles}

Velocity measurements were carried out in several cross sections along the experimental channel length, by means of an electromagnetic velocity meter. Each cross section consists of 12 measuring points. Analysis of the measurements shows that the streamwise velocity reaches its maximum value at the inner bank, right before the apex of the meander bend. For each crosssection, values of the streamwise velocity are higher at the inner bank than at the outer bank, and reach their minimum values at the bottom, as is to be expected. Fig. 2 shows the experimentally measured and numerically computed streamwise velocities for cross-section 17. Comparison shows that the computed velocities agree reasonably well with the measured velocities, only slightly underpredicting the higher values at the top of the section. The numerical velocity profiles are according to the theoretical profiles. Because of technical limitations, the measuring grid for the velocity measurements in this first set of experiments was too coarse to determine a full experimental velocity profile. In a next series of experiments, PIV measurements of the cross sections will be conducted, providing a more accurate representation of the physical velocity profiles. This will allow for a more detailed comparison of the measured and computed velocities, and a better evaluation of the overall accuracy of the numerical computations. 


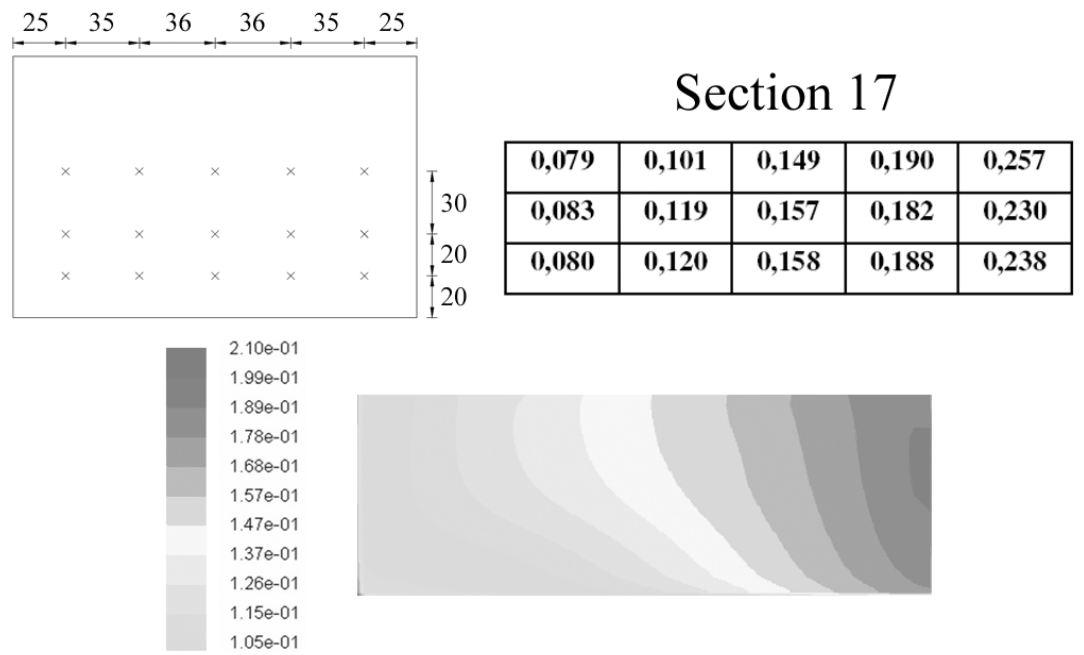

Figure 2: Comparison of experimentally measured and numerically modelled streamwise velocities at cross-section 17. Reported values are in $[\mathrm{m} / \mathrm{s}]$. Upper left frame: distribution of measuring points, dimensions are in $[\mathrm{cm}]$; upper right frame: experimental streamwise velocities measured with the electromagnetic flow meter; bottom frame: contour plot of numerical results.

\subsection{Secondary current}

In order to see whether or not the numerical model captures the motion of the secondary current or transverse flow well, the experiment with $\mathrm{Q}=2.5 \mathrm{l} / \mathrm{s}$ and an outlet height of $5 \mathrm{~cm}$ was repeated. In several sections of the experimental model, streamlines were visualized with pieces of very fine string, suspended near the bottom and near the water surface. This visualization could then be compared with the streamline visualization performed on the numerical output. Comparison yields that both the experimental and the numerical streamlines follow the same path, which is a good first indication that the numerical model captures the motion of the secondary current. The numerical model also allows for cross-sectional vector plots of the secondary current. This way, the output can be compared with the theoretical motion of the secondary current in meander bends, which indicates that the flow consists of a primary rotational cell, and alternates between a clockwise and counter-clockwise motion every other meander bend. Together with this rotational cell, a smaller, counter-rational cell appears near the outer bank (Blanckaert and Graf [11], Blanckaert and De Vriend [12]). 


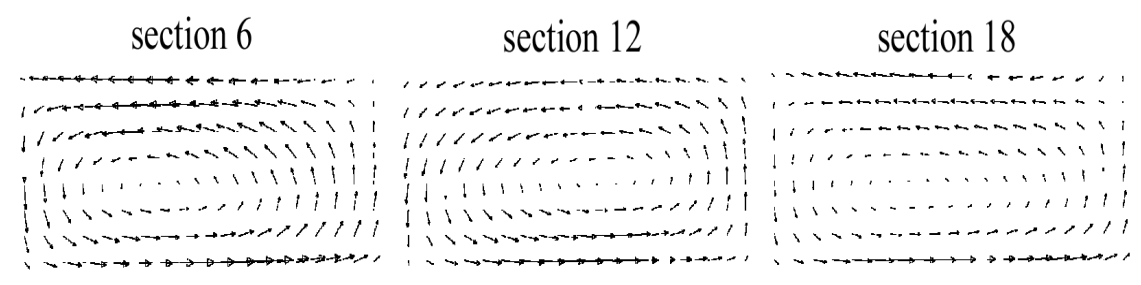

Figure 3: Vector plots of the secondary current. Primary flow direction is into the page. The numbers refer to the sections as defined in Fig. 1.

Fig. 3 shows vector plots of the secondary current in several cross-sections of the three meander bends. It can be seen that there is indeed only one rotational cell, but that the current does not alternate its direction. Moreover, extra numerical simulations with altered geometries show that the direction of the secondary current is dictated by the first meander bend the flow encounters, and that it keeps this direction along the entire model length. In reality, the bathymetry of the deformed river bed in between river bends dissipates the motion of the secondary current in such a way that but a fraction of its strength remains, if not completely disappears. Therefore, the secondary current has little or no 'motion history' when it reaches the next bend, and it takes on the natural rotation in this bend. However, in the experimental and numerical model, there is no dissipation due to a deformed river bed, neither is there a substantial influence of friction. The section in between the river bends is too short to account for a full dissipation, so that the secondary current still keeps the motion it was given

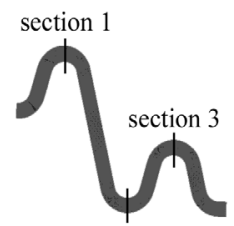

section 2

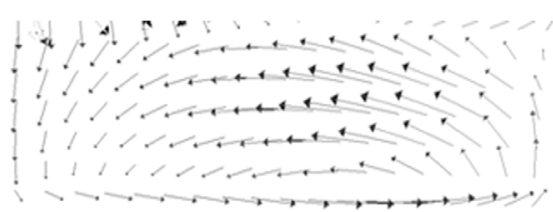

section 1

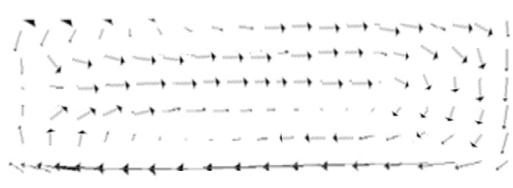

section 2

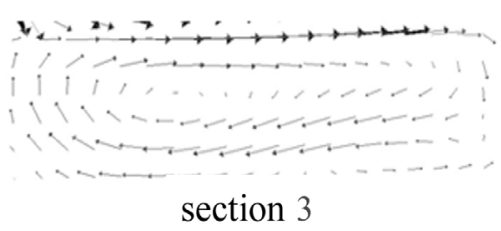

section 3

Figure 4: Upper left frame: geometry with lengthened straight section between the first and second meander bend; upper right frame: vector plot of the secondary current in section 1; bottom frame: vector plots of the secondary current in sections 2 and 3. Primary flow direction is into the page. 
possible explanation, numerical simulations were performed in which the section in between the first and the second meander bend was lengthened to four times in the first bend and resists the natural counter-rotation in the following bends. The motion does however seem to weaken, as can be seen in Fig. 3. To test this the original size, as is displayed in Fig. 4. Also, vector plots are shown of the secondary current in cross sections in the three meander bends. It can be seen that the secondary current indeed switches direction from a counter-clockwise rotation in the first meander bend to a clockwise rotation in the second meander bend. The secondary current does not change rotation in the third meander bend, but keeps the rotation it had in the second bend. Thus, it can be concluded that the short straight sections and the according lack of frictional effects in between the meander bends of the experimental and numerical model are responsible for the described behavior of the secondary current. The appearance of only one rotational cell in the numerical results has two possible explanations: the counter-rotational cell near the outer bank does not exist in our specific experiments, or the numerical grid is too coarse to capture this weaker current. Future measurements should provide more insight into this matter.

\section{Conclusions}

Experimental and numerical models have been developed in order to predict the flow in a meandering river. The model represents two wavelengths of a regime channel. Results are presented for simulations with fixed cross-sections and steady discharge. The main conclusions are: (1) the numerical model accurately predicts the water depth along the channel length and at the outer and inner banks of the meander bends, (2) realistic simulations of the streamwise velocities are produced experimentally as well as numerically and the numerically computed velocities are in good agreement with the experimentally measured velocities, (3) streamline visualisation indicates that the numerical model captures the motion of the secondary current and that the secondary current does not alternate its rotation but maintains the rotation of the first meander bend. In a second set of experiments, the PIV measurement technique will be used to gather velocity data. This will allow for a more detailed comparison of the streamwise velocity patterns, and will enable the visualisation of the secondary current in the experimental model and comparison with the output of the numerical model. Also, a sediment transport module will be incorporated in the numerical model, and experiments with deformable beds will be performed.

\section{Acknowledgements}

This research is supported by research grants from the Flemish Fund of Scientific Research (project nr. G.0330.07). The authors wish to thank the technical staff of the laboratory for their assistance with the experimental testsetup, and Joost Cornelli for his assistance with the numerical computations. 


\section{References}

[1] Olsen, N.R.B., Kjellesvig, H.M., Three-dimensional numerical modeling of bed changes in a sand trap. Journal of hydraulic resources, 37, pp. 189198, 1999

[2] Rajendran, V.P., Constantinescu, S.G., Patel, V.C., Experimental validation of a numerical model of flow in pump-intake bays. Journal of hydraulic engineering, 125, pp. 1119-1125, 1999

[3] Williams, G.P., River meanders and channel size. Journal of hydrology, 88, pp. 147-164, 1986

[4] Rodi, W., Turbulence models and their applications in hydraulics, Balkema, Rotterdam, 1993

[5] Hirt, C. W., Nichols, B.D., Volume of Fluid (VOF) method for the dynamics of free boundaries. Journal of Computational Physics, pp. 201225,1981

[6] Ma, L., Ashworth, P.J., Best, J.L., Elliot, L., Ingham, D.B., Whitcombe, L.J., Computational fluid dynamics and the physical modeling of an upland urban river. Geomorphology, 44, pp. 375-391, 2002

[7] Launder, B.E., Spalding, D.B., The numerical computation of turbulent flows. Computer Methods in Applied Mechanics and Engineering, 3, pp. 269-289, 1974

[8] Nallasamy, M., Turbulence models and their applications to the prediction of internal flows - a review. Comput. Fluids., 15, pp. 1183-1195 , 1987

[9] Patankar, S.V., Numerical heat transfer and fluid flow, Hemisphere, Washington, DC, 1980.

[10] Issa, R.I., Solution of the implicitly discretized fluid flow equations by operator-splitting. Journal of Computational Physics, 62, pp. 40-65, 1985.

[11] Blanckaert K., Graf W.H., Mean flow and turbulence in open-channel bend. Journal of Hydraulic Engineering, 127, pp. 835-847, 2001

[12] Blanckaert K., De Vriend H.J., Secondary flow in sharp open-channel bends. Journal of Fluid Mechanics, 498, pp. 353-380, 2004 